\title{
Market Analysis and Growth Prediction for Online Auctions:A Case Study with Restaurant Certificates
}

\author{
James K. Ho
}

\begin{abstract}
Online auction is one of the few examples of how e-commerce over the Internet can truly transform a business model, rather than simply transplanting it to a different medium. From the selling and buying of personal possessions among private individuals, it has grown to become a major alternative distribution channel for most commercial goods. While restaurants may not be a natural fit for this model initially, viable markets are indeed emerging in the form of gift certificates and discount coupons. In this article, actual development in this approach to internet marketing and advertising in the restaurant industry is surveyed, and a framework for market analysis and growth prediction is presented in the context of market conditions for sellers and buyers. In particular, the methodology of maximum resolution dichotomy is introduced with a case study to analyze data available under the category of Restaurant Gift Certificates on eBay.com.
\end{abstract}

Index Terms-online auctions; market analysis; growth prediction; internet marketing; data mining

\section{INTRODUCTION}

The Internet is a relatively new medium of communication providing unprecedented connectivity among suppliers and consumers of goods and services worldwide. However, the initial hyperbole over its promises led to grossly exaggerated valuations of business models that were mere transplantations of existing processes to the alternative channel, or old wine in new bottles, so to speak. Now that the bubble has burst [11], more sensible and critical thoughts are turning to true transformations that are creating and nurturing markets of the future [12]. Online auction is one of the very few cases that have broken the mold and held a steady course ([9], [10], [14], [15]) as witnessed by the growth of eBay.com. as the entire culture it engenders is now being described as the eBay economy [5]. In Section II we survey the actual development of online auctions in the restaurant sector of the hospitality industries, showing that while it is still in a fledgling stage, viable markets have emerged to allow data-based research and analysis. A methodology to visualize the shape of an online auction market, known as topological analysis [2] is described in Section III. It casts the topological model into a buyer-seller dichotomy in which the dimensions can be partitioned into two groups, each contributing to one part of the buyer-seller dichotomy. Next, the concept and technique of maximum resolution dichotomy ([3], [4]) are applied to interpret the area of each part of the dichotomy as an

James K. Ho, Information \& Decision Sciences, University of Illinois at Chicago, Chicago, USA(email:jimho@uic.edu). aggregate measure of its relative dominance, so that an online auction market at any particular point of its development can be classified as either a buyer's or a seller's market. Samples of data from eBay.com collected from 2003 to 2007 are studied in Section IV. The results are used to predict subsequent market growth and verified with data from 2010 in Section V.

\section{OnLine Auctions For Restaurant Gift CERTIFICATES}

At the height of the dot.com era, businesses of all types and sizes rushed to stake claims on the frontier of cyberspace. Restaurants were no exceptions. Having a Web site and a recognizable domain name on the Internet became de rigor. However, it did not take long for them to realize that restaurants are by nature brick-and-mortar. Driving traffic to Web sites, a major challenge in its own right, may have little to do with bringing customers through the door. By itself, a Web presence is merely an advertisement in a pull, rather than push medium. Build it, and no one may notice. Hooking up with dining guides, classified directories, and search engines can help. But there is no easy and useful measure of the effectiveness of such linkages. So it is little surprise that what appear to have survived after the initial hoopla subsided turn out to be relics of the print medium: gift certificates and discount coupons.

Gift certificates are typically sold at the restaurants, and used as gifts or incentive awards. Discount coupons are mostly distributed freely through printed ads in newspapers and magazines for promotional purposes. As there is cost of production and distribution, enterprising "agents" emerge to publish booklets of discount coupons which they sell for a profit. The featured restaurants honor the discount as a promotional expense but save on direct costs. Customers enjoy the discount at a nominal price. It is this apparent allwin proposition that has found a niche on the Internet. Moreover, instead of a direct transplant of an old business model to a new medium, it has taken on an additional appeal of dynamic pricing, by way of online auctions. The early development of this business model on eBay, from 20012003 was dominated by one major player: restaurant.com (www.restaurant.com). The Arlington Heights, IL-based company serves as both Web host and classified directory for restaurants cost-free in exchange for an obligation to honor some agreed number of gift certificates (typically at $\$ 25$ face value) or discount coupons (typically at $50 \%$ off). It lists and sells such offers online at $\$ 10$ and $\$ 3$, respectively, directly on its own Web site and in eBay auctions. The customer receives a coded certificate by e- 
mail, prints it out and brings to the restaurant where it is validated automatically by phone. As we shall analyze further, the sales strategies of this key player have significant influence in shaping the entire market over the years.

To date, a variety of similar offers by competing agents or restaurants chains have led to a more diverse online auction market on eBay. A sample of completed auctions is shown in Table 1 with the number of bids received and the winning price. For items with no bids, the starting bid is indicated within parentheses. BIN (buy-it-now) marks auctions ended with a direct purchase. On December 1, 2007, there were
1707 active listings on eBay under: Category = Gift Certificates and Type $=$ Restaurant. The comparable number of active listings was 11973 for concerts tickets, 33602 for digital cameras, and 125711 for U.S. coins. While the market under study is perhaps still in a fledgling stage of development, there is already significant data available for formal analysis to track its conditions in terms of whether they are favorable to buyers or sellers. The methodology and empirical results follow.

TABLE I. A SAMPLE OF EBAY AUCTIONS FOR RESTAURANT CERTIFICATES

\begin{tabular}{|c|c|c|}
\hline AUCTION ITEM & BIDS & WIN \\
\hline Seattle WA Tacos Guaymas-\$25 Gift Certificate & $\mathrm{BIN}$ & $\$ 10.00$ \\
\hline \$35 Voucher: Illinois Street Food Emporium in Indiana & 2 & $\$ 15.50$ \\
\hline \$215 Gift Certificate/Coupon OMAHA STEAKS Save $\$ 155.01$ & 2 & $\$ 1.04$ \\
\hline WINGER'S(american diner)\$70 IN PROMOTIONAL CERTIFICATES & 0 & $(\$ 9.99)$ \\
\hline 2 Starbucks FREE drink coupons - No LIMITS - GREAT GIFT & 17 & $\$ 2.89$ \\
\hline \$25 Beto's Gift Card/Gift Certificate -- FREE SHIPPING! & $\mathrm{BIN}$ & $\$ 18.00$ \\
\hline \$100 Starbucks Gift Card w/ Holder & 17 & $\$ 93.00$ \\
\hline \$10 PAPA JOHN'S Gift Card (Virtual) + Promotional code & 5 & $\$ 8.01$ \\
\hline Restaurant.com Gift Certificate $\$ 25$ Coupon ** Free Ship & 8 & $\$ 4.50$ \\
\hline \$50 RESTAURANT.COM Gift Certificate Exp. Nov 07 NR!! & 21 & $\$ 25.00$ \\
\hline Enjoy Dinner for 2: McCormick \& Schmicks $\$ 100$ Value & 2 & $\$ 81.00$ \\
\hline Macaronni Grill, Chilis, VALID IN CALIFORNIA ONLY & 2 & $\$ 14.50$ \\
\hline (15) WENDY'S RESTAURANT "FREE FROSTYS" COUPONS & 7 & $\$ 3.00$ \\
\hline PECORINO RESTAURANT Brentwood CA - \$25 Gift Certificate & 0 & $(\$ 5.50)$ \\
\hline 5 Free PANERA Bagel Bread Packs $\$ 65$ value FREE SH & 10 & $\$ 13.50$ \\
\hline \$200 OLIVE GARDEN gift certificate / card \& RED LOBSTER & 13 & $\$ 180.96$ \\
\hline \$25 RED ROBIN Holiday Gift Card Certificate $+\$ 5$ Bonus & 7 & $\$ 22.78$ \\
\hline$\$ 25$ Outback Gift Card & 0 & $(\$ 25.00)$ \\
\hline Frederick MD Liberty Road Seafood \& Steak-\$25 Gift Cert & $\mathrm{BIN}$ & $\$ 10.00$ \\
\hline Restaurant.com Gift Certificate $\$ 25$ Coupon ** Free Ship & 4 & $\$ 4.00$ \\
\hline 10 ABSOLUTELY FREE WENDYS FROSTY COUPONS NR 1 cent bid & 8 & $\$ 2.50$ \\
\hline \$25 CERTIFICATE TO THOROUGHBRED'S IN MYRTLE BEACH & 8 & $\$ 10.00$ \\
\hline GIFT CERTIFICATES HANA ZEN RESTAURANT SAN FRANCISCO & 0 & $(\$ 39.00)$ \\
\hline CHAPPELLET WINERY Intimate Wine Tasting in NAPA & 1 & $\$ 9.90$ \\
\hline ANAHEIM WHITE HOUSE RESTAURANT GIFT CERTIFICATES & 6 & $\$ 52.68$ \\
\hline
\end{tabular}

\section{TOPOLOGICAL ANALYSIS OF ONLINE AUCTION MARKETS}

In [2], a topological model for an online auction market is defined to be a simultaneous graphical display of all the dimensions of its relevant data base, which provides a geometrical shape as a descriptive, visual statistics of the market. In particular, various dimensions were identified from available data for constructing a specific topological model that can help discern relative advantages to buyers and sellers. Such a model provides a visual cue for whether a market is favorable to buyers or sellers, without expert knowledge of the items involved or the prices attained.

The record of every completed auction is publicly available for a period of two to three weeks. It is kept on eBay in essentially the same format as an active listing.
While numerous dimensions relevant to a buyer-seller dichotomy are conceivable, we focused on identifying those that can be derived from available data.

In [2], twelve dimensions (i.e. attributes) are identified as follows.

1. NET ACTIVITY (auctions with bids)

2. PARTICIPATION (average number of bids per auction)

3. SELLER DIVERSITY (distribution of offers)

4. SELLER EXPERIENCE (distribution of sellers' ratings)

5. MATCHING (auctions ending with a single bid)

6. SNIPING (last minute winning bids)

7. RETAILING (auctions ending with the Buy-ItNow option)

8. BUYER DIVERSITY (distribution of bidder participation) 
9. BUYER EXPERIENCE (distribution of buyers' ratings)

10. DUELING (evidence of competitive bidding)

11. STASHING (evidence of stock-piling)

12. PROXY (use of proxy bidding as evidence of true valuation)

\section{A. Buy-Seller Dichotomy}

To discern whether particular market conditions are favorable to buyers or sellers, we divide the dimensions into a buyer-seller dichotomy. Gross and net activities are significant for inter-market comparisons, and neutral to buyers and sellers. We map gross activities, on some relative scale for all online auction markets, on the upper vertical axis, and use it as the radius of a circle to frame the visual model. Net activities, as percentage of auctions with bids, are mapped on the same vertical axis. Participation, expressed as the average numbers of bids per auction, is mapped on the lower vertical axis. This way, the size of the circle represents the size of the market relative to all others, whereas the span between the net activity and participation indicates its vibrancy.

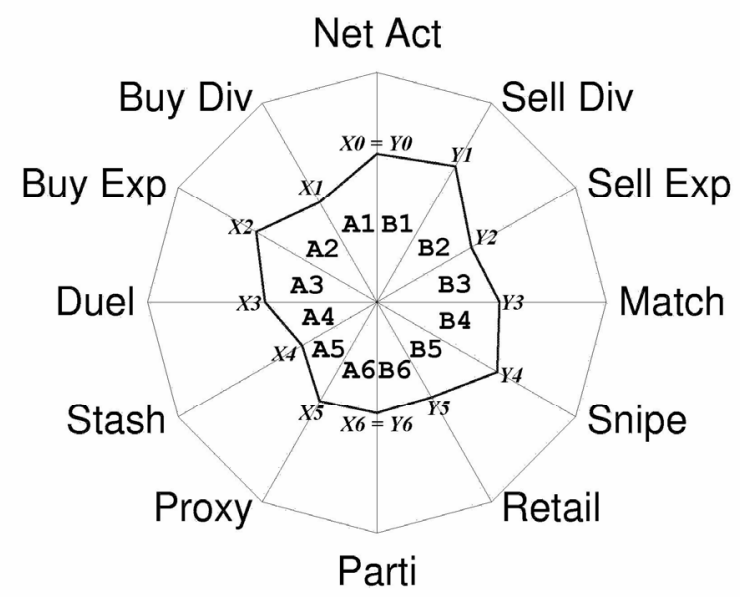

Figure 1. Topological model of online auction market

\section{1) Buyer dimensions}

The seller profiles, in terms of diversity and experience, are buyer dimensions. For diversity, we make the assumption that dominance in market share by relatively few sellers does not provide as much sourcing opportunities for buyers as when there are more different sellers. Analysis of data from over thirty markets led us to propose the use of the proportion of sellers offering 1 per cent or less of the auctions as the metric for diversity. For experience among sellers, the rationale is that an even distribution is favorable to buyers. The skewness of any observed distribution can then be normalized against the even distribution, which is top of the scale. In particular, the maximum of $0.25^{\wedge} 4$ which equals 0.0039 is normalized to 1.0 so that a distribution of, say, $(0.4,0.3,0.2,0.1)$ will be normalized to 0.61 on this scale. The two seller profile dimensions are mapped radially in the top-right quadrant in Figure 1.

Single-bid matching, though not an indicator of the vitality of a true auction market, does work in the favor of buyers. It meets their demand at opening bid prices. The success of winning bids by sniping also put buyers at an advantage. As demonstrated in [13] the winning bid prices can be expected to be undervalued. Buy-It-Now options, if acceptable to buyers, necessarily reflect favorable prices, and so can be ruled a buyer dimension. These three buyer dimensions, all expressed as percentage of total auctions, are mapped in the lower right quadrant in Figure 1.

\section{2) Seller Dimensions}

The buyer profiles, in terms of diversity and experience, are seller dimensions. Analogues to the buyer dimensions we make the assumption that diversity in participation and an even distribution in experience among buyers are favorable to sellers. For diversity, we use the proportion of buyers participating in a single auction as the metric. For experience, the skewness of the distribution is normalized against the even distribution as described above. These two dimensions are mapped in the top right quadrant in Figure

\section{B. Maximum Resolution Topology}

In general, a multi-attribute dichotomy is any multidimensional dataset in which the dimensions can be partitioned into two groups, each contributing to one part of the dichotomy. Given the star glyph [1] of a multi-attribute dichotomy, as exemplified in Figure 1, it will be both visually and intuitively appealing if the areas covered by the two parts can be used as a meaningful aggregate measure of their relative dominance. A larger area on the left side of the glyph means dominance by the left part, and vice versa. In the case of online auction markets, this asymmetry can be interpreted as market conditions being advantageous to either buyers or sellers. In mathematical terms, the aggregate value function takes the form of the sum of pairwise products of adjacent attributes: $\mathrm{V}(\mathrm{X} 1, \ldots, \mathrm{Xn})=\mathrm{C} \Sigma \mathrm{Xi}$ $\mathrm{Xj}$; where attributes $\mathrm{i}$ and $\mathrm{j}$ are adjacent; $\mathrm{Xi}$ is the value of attribute $\mathrm{i}$, for $\mathrm{i}=1, \ldots, \mathrm{n}$; and $\mathrm{C}$ is some scaling constant.

The concept of using the area of the parts of a dichotomy as an aggregate measure of their relative dominance is plausible, since increasing value of an attribute contributes positively to its designated part, as well as the latter's area in the glyph. However, it must be refined to realize its potential, which arises from the degrees of freedom allowed by the topology of the glyph, namely, the configuration of the attributes, and the angles between adjacent pairs thereof. For any given arrangement of the attributes, the standard star plot produces a glyph along symmetrically spaced radial axes. Variations from this symmetry imply a feasible set of shapes and areas, which along with permutations of the configuration, offer the choice of topologies that may suit further criteria for a meaningful aggregate measure function. In particular, we use a diverse subset of the data instances in an optimization model to derive a topology with maximum resolution in discerning dominance with respect to the reference subset [3].

To this end, the first step is to render the glyph unit free by normalizing the data on each dimension to the unit interval $[0,1]$. The second step is to render the glyph context free by harmonizing the dimensions as follows. For each attribute, the quartiles for the values in the entire dataset are computed. A spline function is constructed to map these quartiles into the $[0.25,0.5,0.75]$ points of the unit interval. This way, a hypothetical data instance with all attributes at 
mean values of the dataset will assume the shape of a symmetrical polygon with vertices at the mid-point of each radial axis. In this frame of reference, all shapes and sizes are relative to this generic "average" glyph, and free of either units or specific context of the attributes. For our exploratory work, simple second-order (piecewise linear) splines are used.

\section{Dichotic Dominance with respect to Reference Subsets}

Next, to determine an optimal topology, we use the concept of a reference subset of the data instances to help define dichotic dominance. This concept is best explained in a medical scenario. Suppose a certain disease is monitored by a number of symptoms and tests, with a dichotic prognosis of "life" or "death". Judging from the combination of data for any particular case, it may be difficult to predict. A reference subset is a collection of nontrivial, non-obvious cases with known outcomes, namely life or death. In our exploratory analysis of online auction markets, there is no factual or expert judgment on whether any particular case is a "buyers" or "sellers" market. An initial collection from 34 diverse and well-established markets is used on an ad hoc basis as the reference subset. An arbitrary configuration of the attributes within each part of the dichotomy is selected with the attributes evenly spaced, as in Figure 1. This is analogous to selecting a portfolio of stocks to provide an index for a stock market. The performance of any stock can be gauged relative to the index, which may be arbitrarily chosen initially. With better knowledge of the significance of individual stocks, more useful indices can be established. By the same token, the choice of reference subsets for multi-attribute dichotomies can be adaptively refined as the study progresses.

Once an optimal topology is derived with respect to a given reference subset, any other data instance, an online auction market in our case, can be plotted and visualized as a maximum resolution dichotomy. Moreover, the total enclosed area in the plot, including both parts of the dichotomy may be used as a relative measure of the overall activity of all the attributes. We can consider this as an indicator of the "robustness" of the market. Whereas, the difference in the areas of the left and right parts of the dichotomy provides an index of dichotic dominance among market conditions favoring buyers and sellers. In our settings, a left dominance favors sellers, and a right dominance favors buyers

\section{A Goal Programming Optimization Model}

Subject to the constraints of preserving the prejudged dominance in the reference subset of dichotomies, an optimal topology (configuration of attributes and angles between adjacent pairs) is sought that maximizes the discriminating power, or resolution, as measured by the sum of absolute differences in left and right areas for the reference subset. Such an optimal configuration will be called a maximum resolution topology (MRT). For any given configuration of the attributes, maximization of the discriminating power can be formulated as a linear program (LP). However, LP produces extreme-point solutions, which may reduce some of the angles between attributes to zero, thus collapsing the glyph. To avoid such degeneration, maximization with bounded variation of the angles is modeled as a goal program (GP) in [4].

As exploratory data mining [6], 34 data sets with the bidding records of approximately 500 auctions each are used as the reference subset. The market categories comprise automobiles, business software, camcorders, coffee tables, coins, cordless phones, desktop PCs, digital cameras, gift certificates, handbags, laptop PCs, lodging, necklaces, personal digital devices, rings, travel and vacation packages, and wristwatches. The optimal configuration of the twelve dimensions in the topological model for online auctions as shown in Figure 2.

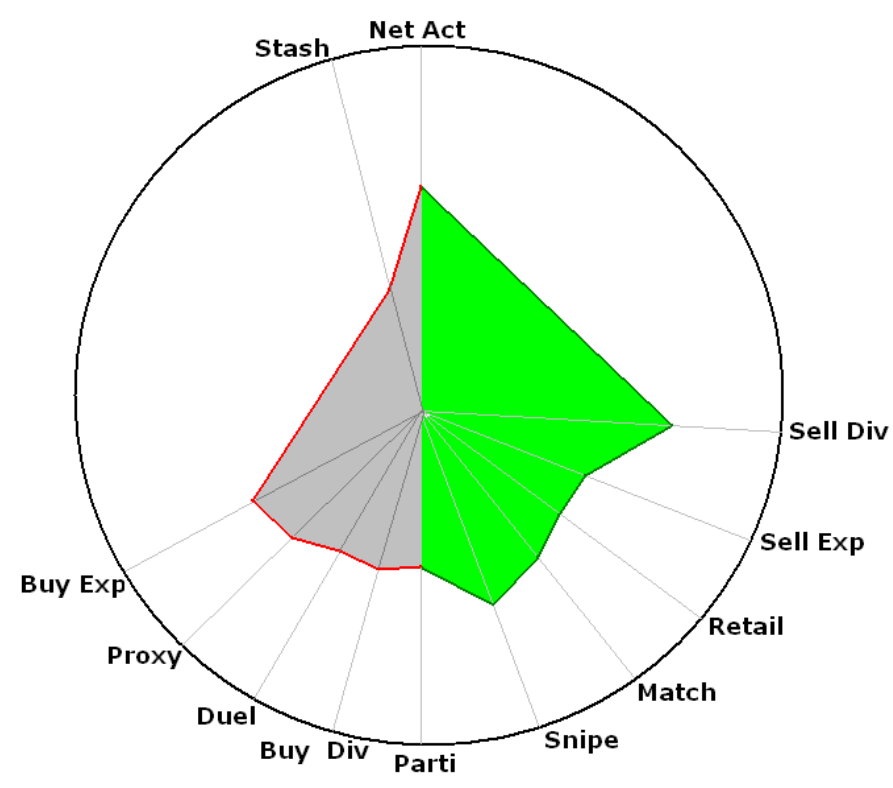

Figure 2. Maximum Resolution Dichotomy for Online Auction Markets

\section{Restaurant CeRtificAtes ON EBAY: 2003 - 2007}

The maximum resolution dichotomy methodology is applied to restaurant certificates on eBay first in Spring 2003. The study was expanded in 2006, and continued in 2007. The specific auction category used was: Gift Certificates $>$ Restaurants. As eBay makes available the bidding history of completed auctions on a 15-24-day rolling basis, MS Excel spreadsheet macro programs were designed using Visual Basic to collect the raw data periodically until the quotas are filled. The target sample size was 500 in each case. The sets for 2006 and 2007 actually comprised five such cases each for further data mining experiment, providing the larger samples for the present work. The data was fed into MS Access databases. Data mining tools in the form of SQL database queries were programmed to extract the information necessary for the topological models. Statistics for the data collected for this study are summarized in Table 2 .

TABLE II. StATISTICS FOR EBAy DATA

$\begin{array}{llll}\text { Year } & \mathbf{2 0 0 3} & \mathbf{2 0 0 6} & \mathbf{2 0 0 7} \\ \text { Sample Size } & 510 & 2740 & 2307\end{array}$




\begin{tabular}{|c|c|c|c|c|c|c|c|}
\hline First Date & $4 / 30$ & $1 / 27$ & $2 / 14$ & Dueling & $35.10 \%$ & $21.06 \%$ & $15.82 \%$ \\
\hline Last Date & $5 / 7$ & $4 / 8$ & $4 / 13$ & Stashing & $1.37 \%$ & $1.25 \%$ & $2.06 \%$ \\
\hline$\%$ with No Bid & 41.3 & 37.3 & 30.8 & Sniping & $6.67 \%$ & $15.97 \%$ & $10.04 \%$ \\
\hline \multirow[t]{2}{*}{ Average \# bids } & 4.49 & 8.11 & 6.69 & BIN & $0.39 \%$ & $84.91 \%$ & $8.30 \%$ \\
\hline & & & & Proxy & $29.22 \%$ & $25.70 \%$ & $23.87 \%$ \\
\hline
\end{tabular}

Offers by Sellers:

$\begin{array}{llll}>10 \% & 11.11 \% & 0.22 \% & 0.38 \% \\ <=10 \% & 0.00 \% & 1.98 \% & 2.10 \% \\ <=3 \% & 11.11 \% & 2.90 \% & 4.72 \% \\ <=1 \% & 77.78 \% & 94.88 \% & 92.80 \%\end{array}$

Rating of Sellers

$\begin{array}{llll}>100 & 55.56 \% & 56.33 \% & 58.31 \% \\ <=100 & 22.22 \% & 27.78 \% & 24.67 \% \\ <=25 & 22.22 \% & 12.06 \% & 11.88 \% \\ <=5 & 0.00 \% & 3.83 \% & 5.14 \%\end{array}$

Participation by Buyers:

$\begin{array}{llll}>10 & 0.00 \% & 1.78 \% & 1.27 \% \\ 5 \text { to } 10 & 1.19 \% & 4.24 \% & 1.89 \% \\ 2 \text { to } 5 & 27.16 \% & 33.67 \% & 22.79 \% \\ 1 & 71.65 \% & 60.31 \% & 74.05 \%\end{array}$

Rating of Buyers:

$\begin{array}{llll}>100 & 24.31 \% & 43.47 \% & 46.55 \% \\ <=100 & 22.57 \% & 29.51 \% & 27.70 \% \\ <=25 & 22.29 \% & 18.69 \% & 15.73 \% \\ <=5 & 30.83 \% & 8.33 \% & 10.02 \%\end{array}$

After appropriate normalization and harmonization, the complete data for the three cases are plotted in the maximum resolution topology (MRT) derived from the reference set. The resulting maximum resolution dichotomies (MRD) are shown in Figure 3 and revealed visually that the left side area dominates the left in 2003, indicating that they were relatively favorable to sellers. However, in 2006 and 2007, the right side area dominates the left, suggesting a buyer's market by our functional definition in topological analysis. As quantitative measures, we can use an index:

$$
\mathrm{I}_{\mathbf{B}}=(\text { Right Area - Left Area }) /(\text { Right Area + Left Area })
$$

Which is positive for a buyer's market, and negative for a seller's market. This index ranges from -0.29 to +0.43 with an average of 0.11 for the 34 cases in the reference set. For restaurant certificates, we have the indices in Table 3.

TABLE III. ONLINE AUCTION MARKET INDICES
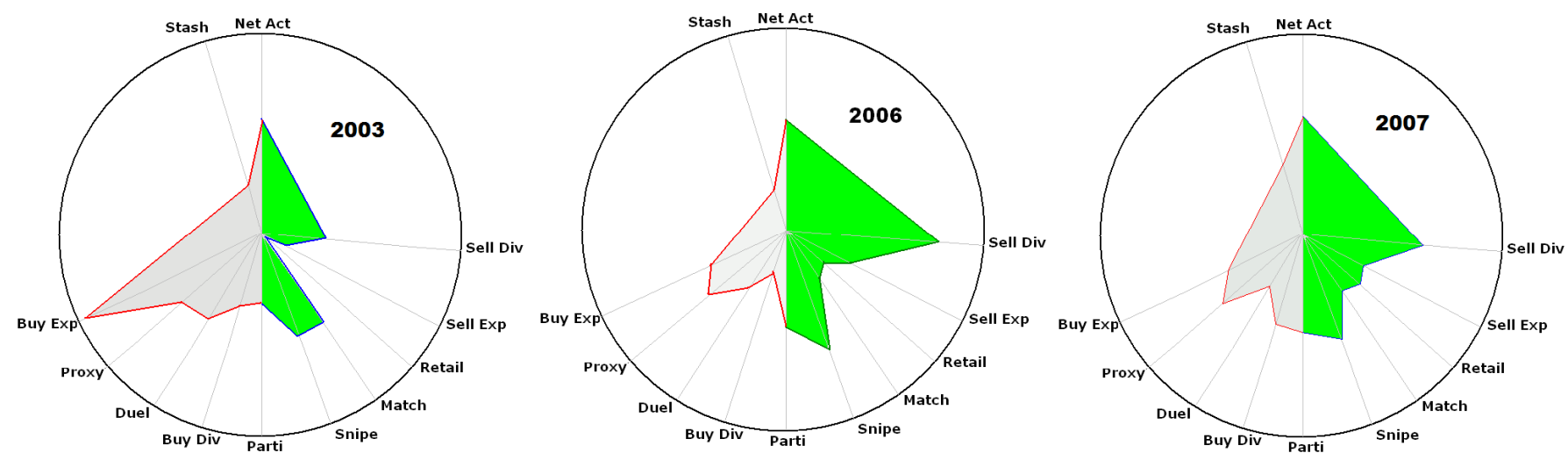

Figure 3. . Maximum Resolution Dichotomies for Restaurant Certificates

\section{PREDICTION AND VERIFICATION OF MARKET GROWTH}

From our reference set used to derive a maximum resolution topology for online auction markets, we observe that conditions on eBay are generally favorable to buyers. That is logically consistent with its phenomenal and sustained growth because it is buyers that pay for the markets. In particular, restaurant certificates in 2006 and 2007, with indices of 0.5 and 0.44 , respectively, are well above average. The changing shape of the online auction market for restaurant certificates can be described and explained to further depth with additional observation of the 
sales strategies of its key player: restaurant.com. In 2003, this company listed all its offers on eBay as regular auctions with no provision for direct purchase (BIN). Being the largest of only a handful of sellers ( 9 in our sample) at the time, it dominated the market (accounting for 488 of the 510 auctions), attracting buyers with a very even mix of experience. Overall, it was a seller's market [7]. Subsequently other, less experienced sellers entered the market increasing the diversity of supplies. At the same time, restaurant.com offered the BIN option making it more convenient for buyers who did not want to wait out an auction. Perhaps sensing the popularity of BIN and the high proportion of single bid auctions, restaurant.com eventually moved its listings out of eBay auctions to its eBay store, which is structured more simply for online retail sales. This switch, along with the entry of more new sellers, accounted for the significant change on the right-side of the plots between 2003 and 2006. The market (as online auction, rather than retail) was then quite favorable to buyers. In 2007, restaurant.com resumed its listings on eBay auctions but as BIN only (and not auctions with a BIN option). This had a distorting effect on the shape of the auction market and should perhaps be excluded in future studies. Though it has moved away from bona fide online auctions, its sales gave rise to a secondary source of supply for the market. For promotional purposes, it offers occasional and selective discounts on its prices of $\$ 25$ for certificates and $\$ 3$ for discount coupons. This provides potential leverage for resellers who can then list the certificates on eBay and hope to make a profit from the price differentials. Finally, we observe that as eBay continues to expand and gain new users, the buyers of restaurant coupons tend to be relatively experienced. In other words, it is fair to say that few users begin their journey on eBay looking for good deals on restaurants. As the market appears to remain favorable to buyers, it is not clear if there is sufficient motivation for sellers to invest in its sustained growth [8]. For these reasons, we predicted in 2007 that this particular market may not follow the generally positive growth path expected of the eBay.com online auction platform. This observation is at least partially verified with incidental data in 2010 as summarized in Table 4. Using our methodology of topological analysis, we hope to track and project such trend as we continue our study.

TABLE IV. COMPARATIVE GROWTH OF ONLINE AUCTION MARKETS

Active Listings on eBay.com
Gift Certificates $>$ Restaurant
Concert tickets
Digital cameras
U.S. coins

\section{ACKNOWLEDGEMENT}

Yibin Xiao designed the MS Excel macro programs used in the data mining and analysis in this work.

\section{REFERENCES}

[1] J. Chambers, W. Cleveland, B. Kleiner and P. Tukey, Graphical Methods for Data Analysis, Belmont, CA: Wadsworth Press ,1983..

[2] Ho, James K. (2004) 'Topological analysis of online auction markets', International Journal of Electronic Markets 14(3): 202-213.

[3] J.K. Ho and S.C.K. Chu, "Maximum resolution topology for multiattribute dichotomies" Informatica, vol.16, no. 4,2005, pp. 557-570.

[4] J.K. Ho, S.C.K. Chu. And S.S. Lam (2007) "Maximum Resolution Topology for Online Auction Markets", International Journal of Electronic Markets, vol 17, no. 2, 2007, pp. 164-174.

[5] R.D. Hof, "The eBay economy", BusinessWeek, August 25, 2003.

[6] P. Hoffman and G. Grinstein, "A survey of visualizations for highdimensional data mining", in Usama Fayyad et al (eds.) Information Visualization in Data Mining and Knowledge Discovery, Morgan Kaufmann, 2001, pp 47-82.

[7] D. Houser and J. Wooders, "Reputation in auctions: theory, and evidence from eBay", Journal of Economics and Management Strategy, vol. 15, no. 2, 2006, pp. 353-269.

[8] M. Kamins, X. Dreze and V. Folkes, "Effects of seller-supplied prices on buyers' product evaluations: Reference prices in an Internet auction contextt", Journal of Consumer Research, vol. 30, no. 4, 2004, pp. 622-628.

[9] S. Klein, "Introduction to electronic auctions," International Journal of Electronic Markets, vol. 7, no. 4, 1997, pp. 3-6.

$\begin{array}{rrr}\mathbf{1 2} / \mathbf{1} / \mathbf{2 0 0 7} & \mathbf{9 / 1 8 / 2 0 1 0} & \text { \%change } \\ 1707 & 407 & -76.16 \% \\ 11973 & 49916 & 316.90 \% \\ 33602 & 65210 & 94.07 \% \\ 125711 & 465609 & 270.38 \%\end{array}$

[10] D. Lucking-Reiley, “Auctions on the Internet: What's being auctioned, and how?" The Journal of Industrial Economics Vol. 48, 2000, pp. 227-52.

[11] A.B. Perkins. and M.C. Perkins, The Internet Bubble (rev. edn), New York: HarperBusiness, 2001.

[12] M. Rappa, "Business models on the Web", Managing the Digital Enterprise, http://digitalenterprise.org/models/models.html, 2003.

[13] A.E. Roth and A. Ockenfels, "Last minute bidding and the rules for ending second-price auctions: theory and evidence from a natural experiment on the Internet", American Economic Review, vol.92, no. 4, 2002, pp. 1093-1103.

[14] E. Turban, "Auctions and bidding on the Internet: An assessment" International Journal of Electronic Markets, vol.7, no. 4, 1997, pp. 7-11.

[15] J.K. Ho, "Online auctions: Pragmatic survey and market analysis", In In Lee (Ed.), Encyclopedia of E-Business Development and Management in the Global Economy. Hershey, PA: IGI Global, 2010, pp. $418-427$. 